\title{
Rediseño e Implementación de Un PRototipo PARA LA MEDICIÓN Y CONTROL DE NIVEL Y CAUDAL DE AGUA
}

\author{
José LeONARdo Ramírez EchaVARRía ${ }^{1}$ \\ Carlos Mario Londoño Parra ${ }^{2}$
}

\section{Resumen}

Este artículo presenta el proceso de rediseño de una planta de medición de nivel y caudal, como un prototipo didáctico, incorporando diferentes tecnologías de medición. Se incluyen diferentes estrategias de control para una misma variable y la posibilidad de comparar los resultados de las mediciones realizadas.

En la ejecución del proyecto, se reubicó parte de la instrumentación existente y se instalaron nuevos equipos. Se calibraron los equipos que lo requerían, se rediseñó el tablero eléctrico y se actualizaron los planos de cableado, permitiendo articular el sistema de control con la instrumentación de campo con la que se dotó el sistema. Finalmente, se realizaron las respectivas pruebas técnicas y se elaboraron las guías de laboratorio para la medición y control de nivel y caudal de agua. No obstante, se deja la posibilidad de expandir el prototipo didáctico mediante la implementación de nuevas estrategias de control tales como SCADA y otros sistemas de adquisición de datos que permitan el desarrollo de aplicaciones de simulación.

1 Ingeniero Electricista. Universidad de Antioquia.Especialista en Gestión Energética Industrial, ITM. Especialista en Automática, UPB. MSc(c). en Ingeniería Énfasis en Automática, UPB.

2 Ingeniero Electricista, Universidad de Antioquia. Especialista en Automática, UPB. MSc(c). en Ingeniería Énfasis en Automática, UPB. 


\section{Palalures clave}

Transductor, control lógico programable, calibración, control de nivel, control de flujo, instrumentación, prototipo didáctico.

\section{ABstract}

This article aims to show the redesign process of a plant for level and flow control that includes different technologies of measurement. The plant operates as a didactic dispositive. This project includes different control strategies so measurement results can be compared.

A part of the existing instrumentation was relocated and new equipment was installed. The equipment that required fine tuning was calibrated. The electric board was redesigned and the wiring plans were updated so that the control system was articulated with the field instrumentation given to the system. Finally, relevant technical trials were carried out, and laboratory guides regarding the measure and control of water level and volume were developed. Additionally, the possibility to expand the didactic prototype by means of new control strategies implementation was included in the final recommendations. Such strategies include SCADA and systems of data acquisition in order to develop applications to simulate the system.

\section{Key words}

Transducer, programmable logic control, calibration, level control, flow control, instrumentation, didactic prototype. 


\section{InTRODUCcIóN}

Las tendencias modernas del control cada día aprovechan más la tecnología del computador, así como el control lógico programable para realizar simulaciones de procesos, recolección y almacenamiento de datos, que luego son consultados y utilizados en la elaboración de reportes que permitan hacer seguimientos históricos de las pruebas realizadas y tomar las mejores decisiones en un proceso de producción industrial. [Grupo Tar, 2003]

Con el propósito de implementar un moderno prototipo de medición de nivel y caudal de agua que esté a la altura de laboratorios de alto nivel universitario, el grupo de investigación GINVESTAP del ITM, como parte del proyecto de investigación "Rediseño e implementación de un prototipo para la medición y control de nivel, presión y caudal en el laboratorio de Fluidos" decide mejorar el laboratorio de fluidos dotándolo de instrumentación de última tecnología, esto es, aprovechando los recursos existentes y adquiriendo nuevos equipos.

Los instrumentos más relevantes del prototipo didáctico, incluyendo la instrumentación existente son los siguientes: un controlador lógico programable (PLC), dos tanques, sensores magnéticos para el control del nivel de punto, un variador de velocidad vinculado a una bomba centrífuga para el control de flujo, una válvula mariposa con posicionador y actuador neumáticos $\mathrm{y}$ su convertidor de presión a corriente, un rotámetro visualizador de flujo, interruptores de flujo para la protección de las bombas, un indicador de nivel magnético tipo by pass, una celda de presión diferencial instalada en el fondo del tanque metálico, para medición de nivel por el sistema de brazo húmedo, una celda de presión manométrica instalada por debajo del tanque acrílico, para medición de nivel por presión hidrostática. Adicionalmente, cuenta con un transmisor de presión diferencial, ubicado en la red de tuberías de salida del tanque de acrílico. Este transmisor está asociado a una placa de orificio, y fue concebido para realizar prácticas de flujo poniendo en circulación el agua entre los dos tanques. 
Con la realización de este prototipo didáctico, el ITM dispone de un sistema completo para la realización de prácticas que propician la formación de profesionales capaces de analizar procesos y seleccionar instrumentos de medición y control de aplicación industrial o de manufactura, utilizando la más sofisticada y moderna tecnología existente en el campo y que respondan a las necesidades del sector productivo.

\section{Medición de PREsión}

En este proyecto se utiliza la presión para la medición indirecta del nivel y flujo de agua, pues ésta puede llegar a tener efectos directos o indirectos en el valor de las variables del proceso.

La presión puede definirse como una fuerza por unidad de área o superficie. [Ica, 2001]

Tenemos que:

$$
P=\frac{F}{A}
$$

Donde:

$F$ es la fuerza aplicada perpendicularmente sobre la superficie $[\mathrm{N}]$.

$A$ es el área de la superficie $\left[\mathrm{m}^{2}\right]$.

$P$ es la presión $[\mathrm{Pa}]$.

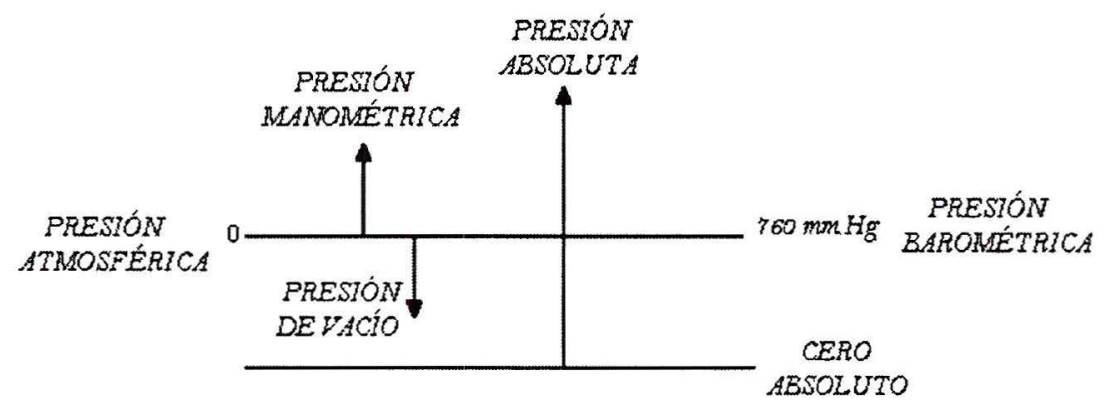

Figura 1. RELACIÓN ENTRE DIFERENTES TIPOS DE PRESIÓN 


\section{Medición de nivel}

El nivel de un líquido contenido en un recipiente se determina midiendo la distancia desde su superficie a una línea de referencia [M Squared_LTD, 1990]. Esta línea constituye el punto de partida para la medición de nivel. La línea de referencia se puede ubicar en cualquier punto a lo largo de la altura del líquido, siendo el punto más común el fondo del tanque.

Algunos factores que influyen en la medida de nivel, son:

- El rango de medición

- La naturaleza del fluido

- Las condiciones de operación

- El tipo de medición requerida

- Las variables de proceso, como la presión y la temperatura

Se aprovechan los transmisores de presión instalados en el fondo de ambos tanques para determinar la presión hidrostática ejercida por el agua contenida en ellos, y mediante la ecuación (4) se puede conocer el nivel [M Squared_LTD, 1990].

$$
h=\frac{P}{\rho g}
$$

Donde:

$P$ es la presión ejercida por el agua $[\mathrm{Pa}]$

$\rho$ es densidad del agua $\left[\mathrm{kg} / \mathrm{m}^{3}\right]$

$h$ es la altura del agua [m].

\section{MeDICIÓN DE FLUUo}

Existen cuatro razones importantes para utilizar sistemas de medición de flujo, éstas son:

- Control de inventarios de producto

- La evaluación del funcionamiento

- La investigación y desarrollo de nuevas tecnologías

- El control de procesos 
La medida de caudal en conducciones cerradas, consiste en determinar la cantidad de masa o volumen que circula por la conducción por unidad de tiempo, mientras que la medición de flujo consiste en determinar la velocidad del fluido a través de una tubería [Squared, 1990].

Como se menciona en [Squared, 1990] "Los instrumentos de medición de caudal se denominan, habitualmente, caudalímetros o medidores de caudal, constituyendo una modalidad particular los contadores, los cuales integran dispositivos adecuados para medir el volumen que ha circulado por la conducción. Los instrumentos que miden flujo se denominan flujómetros".

Los medidores de caudal volumétrico pueden determinar el caudal de volumen de fluido de dos formas: Medición directa, mediante dispositivos de desplazamiento positivo, o medición indirecta, mediante dispositivos de presión diferencial, área variable, velocidad, fuerza, entre otros. [Ordoñes, 2005].

Empleando el transmisor de presión diferencial ubicado en la red de tuberías, y la placa de orificio, ${ }^{3}$ se puede determinar indirectamente, bien sea el flujo o el caudal volumétrico de agua, mediante el empleo de las ecuaciones (2) y (3), respectivamente. Éstas resultan de combinar la ecuación de continuidad y la ecuación de Bernoully [Gutierrez, 2003].

$$
\begin{aligned}
& V_{2}=\left(\sqrt{\frac{2 g}{1-\frac{A_{2}^{2}}{A_{1}^{2}}}}\right)\left(\sqrt{\frac{\Delta P}{\rho}}\right) \\
& Q=A_{2}\left(\sqrt{\frac{2 g}{1-\frac{A_{2}^{2}}{A_{1}^{2}}}}\right)\left(\sqrt{\frac{\Delta P}{\rho}}\right)
\end{aligned}
$$

3 Instalando la placa de orificio dentro de la tubería, se genera la diferencia de presiones, debido a la reducción momentánea del área por donde circula el fluido. 
Donde:

$\Delta P=P_{1}-P_{2}$ es presión diferencial medida por el transmisor, en la tubería y en la obstrucción $[\mathrm{Pa}]$

$\rho$ es densidad del agua $\left[\mathrm{kg} / \mathrm{m}^{3}\right]$

$A_{1}$ es área de la tubería $\left[\mathrm{m}^{2}\right]$.

$A_{2}$ es área del orificio de la placa $\left[\mathrm{m}^{2}\right]$.

$g$ es la fuerza de gravedad $\left[\mathrm{m} / \mathrm{s}^{2}\right]$.

$V_{2}$ es el flujo del agua a través de la placa $[\mathrm{m} / \mathrm{s}]$.

$Q$ es el caudal volumétrico $\left[\mathrm{m}^{3} / \mathrm{s}\right]$

\section{IMPLEMENTACIÓN DEL PROTOTUPO}

Antes de exponer las modificaciones y las innovaciones realizadas al prototipo, se describen algunos apartes del estado del laboratorio en su condición original y las razones que motivaron hacer las reformas.

Este laboratorio fue dotado de algunos recursos y equipos a mediados del año 2000, y fue diseñado para realizar prácticas de medición de nivel y caudal de agua. Algunos de los problemas que tenía, eran los siguientes:

- Las tomas de presión de la placa de orificio fueron ubicadas por debajo del transmisor de presión diferencial, en consecuencia, quedaba un espacio de aire en los conductos del transmisor impidiendo que el agua los llenara completamente entre operaciones consecutivas del sistema. En estas condiciones la densidad del fluido cambiaba, por ser una mezcla de aire y agua, y se requería estar purgando los conductos para que se llenaran completamente de agua. Además esta circunstancia incidía directamente en la repetitividad de la medida, implicando una pérdida de tiempo en la puesta en marcha de la práctica siguiente, debido a la purga del transmisor.

- Se contaba con un obsoleto PLC, con serias dificultades para que el programador implementara la comunicación con otros dispositivos y desarrollara algoritmos de control como PID. Con 
este PLC era difícil realizar las actividades de control prácticoexperimentales programadas en el desarrollo curricular de los núcleos relacionados al control continuo.

- Los transmisores de presión estaban descalibrados, lo que permitía la introducción de errores en la medida de las variables.

- El tablero de control carecía de planos y no cumplía con las normas de seguridad ni con las normas propias de ejecución de esquemas eléctricos, dificultando la intervención en él y, además, su uso se hacía peligroso.

Por las razones descritas en los párrafos anteriores, las prácticas de control de nivel y flujo no se realizaban.

Después de la intervención del sistema, se redistribuye la red de tuberías de interconexión entre los tanques, se rediseña el tablero de control y se instalan nuevos instrumentos de medición, adquisición de datos y control de las variables. Se integran todos éstos en una unidad para obtener la planta piloto, la cual recircula agua de un tanque al otro a través de las tuberías. En esta planta se ejecutan diferentes estrategias de control convencional, mediante la implementación de diversos lazos de control para una misma variable (ver la tabla 1) y se confrontan las medidas obtenidas por los diferentes instrumentos.

La gestión de control en el nuevo prototipo se puede llevar a cabo implementando diferentes rutinas de control en el PLC, o mediante el controlador de procesos de propósito general, el cual dispone de entradas y salidas análogas.

\subsection{Reformas realizadas al sistema existente}

\subsubsection{Reuhicación de la placa de orificio y el transmisor de presión}

El transmisor de presión se ubicó por debajo de la placa de orificio, de este modo se evita recalibración del transmisor, se 
mejora la repetividad de la medida y se evita la pérdida de tiempo, ocasionada por el drenaje continuo del transmisor.

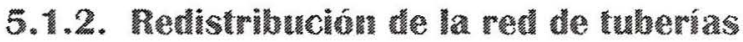

Se instaló un desagüe en el tanque metálico (véase la figura 2) el cual permite, por un lado, desocupar completamente el tanque sin necesidad de accionar la bomba; por el otro, reduce el consumo de energía y de mantenimiento.

Se reubicó la tubería de 2 pulgadas que conduce el agua del tanque metálico al tanque de acrílico. La tubería de 2 pulgadas se trasladó 50 centímetros hacia la pared, dejando espacio para ubicar el tablero de control y el computador.

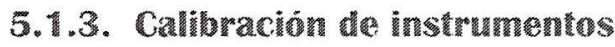

Se calibraron los instrumentos existentes y los nuevos, tomando como instrumentos de comparación, el rotámetro para la medida de flujo y un flexómetro con resolución de milímetros para el nivel de agua [Scheex].

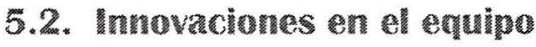

\subsubsection{Semsor de mivel magnético, tecnología bywass}

Se instaló un indicador de nivel en el tanque metálico que funciona con base en el principio de vasos comunicantes, y dispone de un flotador magnético que actúa sobre unas banderas magnéticas que cambian de color indicando el nivel en forma continua a medida que se desplaza el flotador. Además, tiene la ventaja de poder efectuar control de nivel discreto, mediante dos interruptores magnéticos, también accionados por el recorrido del flotador al cambiar el nivel. Se emplea este sistema para control de máximo y mínimo nivel.

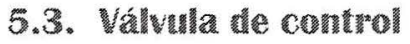

Se instaló una válvula proporcional en la tubería de 2" para control de flujo. El lazo de control para este elemento final, inicia 
con una salida de corriente análoga de 4-20 mA., que puede provenir tanto del controlador de propósito general como del PLC. La selección del controlador se efectúa desde el tablero de control mediante un interruptor de dos posiciones ON-OFF. La salida de 4-20 mA., la recibe un convertidor de presión corriente a presión $(I / P)$ y la entrega a un posicionador que se encarga de dar una salida controlada de presión a un actuador superpuesto sobre la válvula para regular su apertura en función de la señal de corriente.

Las entradas de ambos controladores están conectadas en serie a una señal de 4-20 mA., que proviene del transmisor de flujo de pulsos. Este último recibe una frecuencia de pulsos del proceso, mediante un sensor de paletas.

\subsection{Sensor de palezas rotativas para medición de flujo}

Otra posibilidad de control de flujo se puede conseguir con el sensor de flujo de paletas rotativas, el cual ofrece a los practicantes del laboratorio otra una nueva alternativa de control. Antes, sólo se recibía la señal de flujo proveniente de la placa de orificio y el transmisor, ahora con este sensor de paletas, se pueden estudiar y confrontar nuevas aplicaciones de medición de flujo, basadas en esta tecnología.

\subsection{Rotámetro}

Se instaló un sensor de flujo de área variable, que puede ser usado como instrumento de verificación de flujo contrastando contra él los resultados de las mediciones de la placa de orificio y del sensor de paletas rotativas.

\subsection{Interruptores de flujo}

Estos interruptores de flujo permiten brindarle al sistema una seguridad para que las bombas no operen en vacío, evitando daños en ellas, debido a la inexperiencia de los practicantes o al desconocimiento del funcionamiento del sistema. 


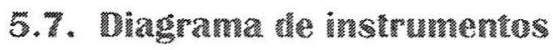

En la figura 2, se presenta el diagrama de instrumentos completo del prototipo [Isa, 1984], [Kauffman, 1998]. Este diagrama se puede desagregar para obtener lazos de control particulares, seleccionando los instrumentos de medición y control apropiados, tomando como referencia la tabla 1.

\subsection{Posibuilinates the controw}

En la tabla 1 se presenta un resumen de las aplicaciones que se pueden implementar, en el prototipo de medición y control de variables de flujo y nivel de agua.

Por ejemplo, un posible lazo de control para una aplicación específica de control de flujo puede emplear el sensor de flujo tipo turbina (FE) que envía una frecuencia de pulsos por unidad de volumen a un transmisor de flujo (FTI - 2). Este transmisor convierte la señal de pulsos en una salida análoga de 4-20 mA., la cual reciben tanto el PLC como el controlador de propósito general (FCI). El elemento final de control puede ser la válvula proporcional. (Ver la tabla 1 y la figura 2)

Otro posible lazo de control, se puede establecer utilizando los mismos elementos primarios y los controladores descritos en el párrafo antexior, y sustituyendo el elemento final por el variador de frecuencia (FY).

El PLC puede recibir la salida de pulsos en función del volumen (mediante un relé de estado sólido) del transmisor (FTI - 2) para hacer control de baches, y establecer otros posibles lazos de control, empleando la válvula proporcional o el variador de velocidad, como elementos finales de control. Similarmente, se pueden obtener otros lazos de control para medición de nivel, combinando los instrumentos, de acuerdo a las posibilidades planteadas en la tabla 1 . 


\section{CONCLUSIOnes}

El control de flujo de agua con la válvula mariposa (la cual presenta un movimiento rotativo del vástago) es una labor ardua, dada su característica de apertura rápida. Al realizar las pruebas se encontró que un buen control de flujo se logra para una apertura de la válvula entre 20 y 60 grados, por fuera de este rango el sistema pierde estabilidad. Otras válvulas de movimiento lineal del vástago (como la válvula globo, por ejemplo) puede mantener la variable controlada en un rango mucho mayor que la mariposa. Sin embargo se optó por una válvula mariposa, por su bajo costo, peso y espacio requerido para su instalación.

Una de las etapas más críticas del prototipo fue la selección de la instrumentación, de tal forma que se ajustara a los rangos de operación de la planta existente, y que cumpliera la doble función de servir a las prácticas académicas y se proyectara a las aplicaciones reales que se presentan en la industria.

E1 prototipo construido integra diferentes tecnologías de medición y control de nivel y caudal, y representa para los estudiantes una gran oportunidad de tener un contacto físico y entrenamiento académico para desarrollar aplicaciones similares en el campo laboral.

Este proyecto es un prototipo a mediana escala, que cumple con todas las especificaciones técnicas de un control de nivel y caudal aplicado al sector productivo.

\section{RECOMENDACIONES}

El prototipo cuenta con una pantalla de operador instalada y conectada al PLC. Se propone para trabajos futuros, desarrollar una aplicación SCADA (adquisición de datos, supervisión y control) que permita monitorear el proceso y obtener datos de las variables para la modelación del sistema.

Se plantea implementar otros sistemas de adquisición de datos, para abrir el portafolio de posibilidades de control y desarrollar 
aplicaciones de simulación tanto en tiempo real, como fuera de línea, mediante la consulta de los datos históricos guardados en disco, adquiridos por el scada. Para este fin, se puede aprovechar el "Matlab" y el "Simple Control" (software aplicativo del control).

En un futuro cercano se puede formular un proyecto que puede ser apoyado por algunos estudiantes del semillero de investigación en automatización de procesos GINVESTAP del ITM, con la intención de desarrollar un control de nivel, empleando la tecnología de burbujeo en el tanque, y aprovechar la toma de aire disponible a la salida de la unidad de mantenimiento que permita experimentar y estudiar esta técnica de control de nivel.

Se recomienda expandir las posibilidades del controlador de propósito general, para hacer control de nivel en tanque abierto con brazo húmedo y control de nivel máximo y mínimo. El control debe ser automático, empleando la lógica cableada y los relés de reserva disponibles en el tablero de control.

\begin{tabular}{|c|c|c|c|c|c|}
\hline \multirow{2}{*}{\multicolumn{2}{|c|}{$\begin{array}{l}\text { LAZO DE CONTROL Y } \\
\text { DISPOSITIVO }\end{array}$}} & \multicolumn{4}{|c|}{ APLICACIÓN } \\
\hline & & \multirow{2}{*}{$\begin{array}{c}\text { NIVEL } \\
\text { CONTINUO } \\
\text { (TANQUE ABIERTO) } \\
\mathrm{X} \\
\end{array}$} & \multirow{2}{*}{$\begin{array}{l}\text { NIVEL CONTINUO } \\
\text { (BRAZO HÚMEDO) }\end{array}$} & \multirow{2}{*}{$\begin{array}{l}\text { NIVEL DE PUNTO } \\
\text { MÁXIMO-MÍNIMO } \\
\mathrm{X}\end{array}$} & \multirow{2}{*}{ Fuwo } \\
\hline \multirow{7}{*}{ SENSOR } & LTI - 1 & & & & \\
\hline & LTI -2 & & $\mathrm{X}$ & $\mathrm{X}$ & \\
\hline & FTI - 1 & & & & $\mathrm{X}$ \\
\hline & $\mathrm{FE}$ & & & & $\mathrm{X}$ \\
\hline & $\begin{array}{l}\text { LSL, } \\
\text { LSH - } 1\end{array}$ & & & $\mathrm{X}$ & \\
\hline & LISHL & & & $\mathrm{X}$ & \\
\hline & FI & & & & * \\
\hline \multirow{2}{*}{ CONTROLADOR } & PLC & $\mathrm{X}$ & $\mathrm{X}$ & $\mathrm{X}$ & $\mathrm{X}$ \\
\hline & $\mathrm{FCI}$ & $* *$ & $* *$ & $* *$ & $\mathrm{X}$ \\
\hline \multirow{2}{*}{ ACTUADOR } & FV & $\mathrm{X}$ & $\mathrm{X}$ & & $\mathrm{X}$ \\
\hline & FY & $\mathrm{X}$ & $\mathrm{X}$ & $\mathrm{X}$ & $\mathrm{X}$ \\
\hline
\end{tabular}

* Solo se puede visualizar 
Figura 2. Diagrama de INSTRUMENTOS DEL PROTOTIPO DIDÁCTICO

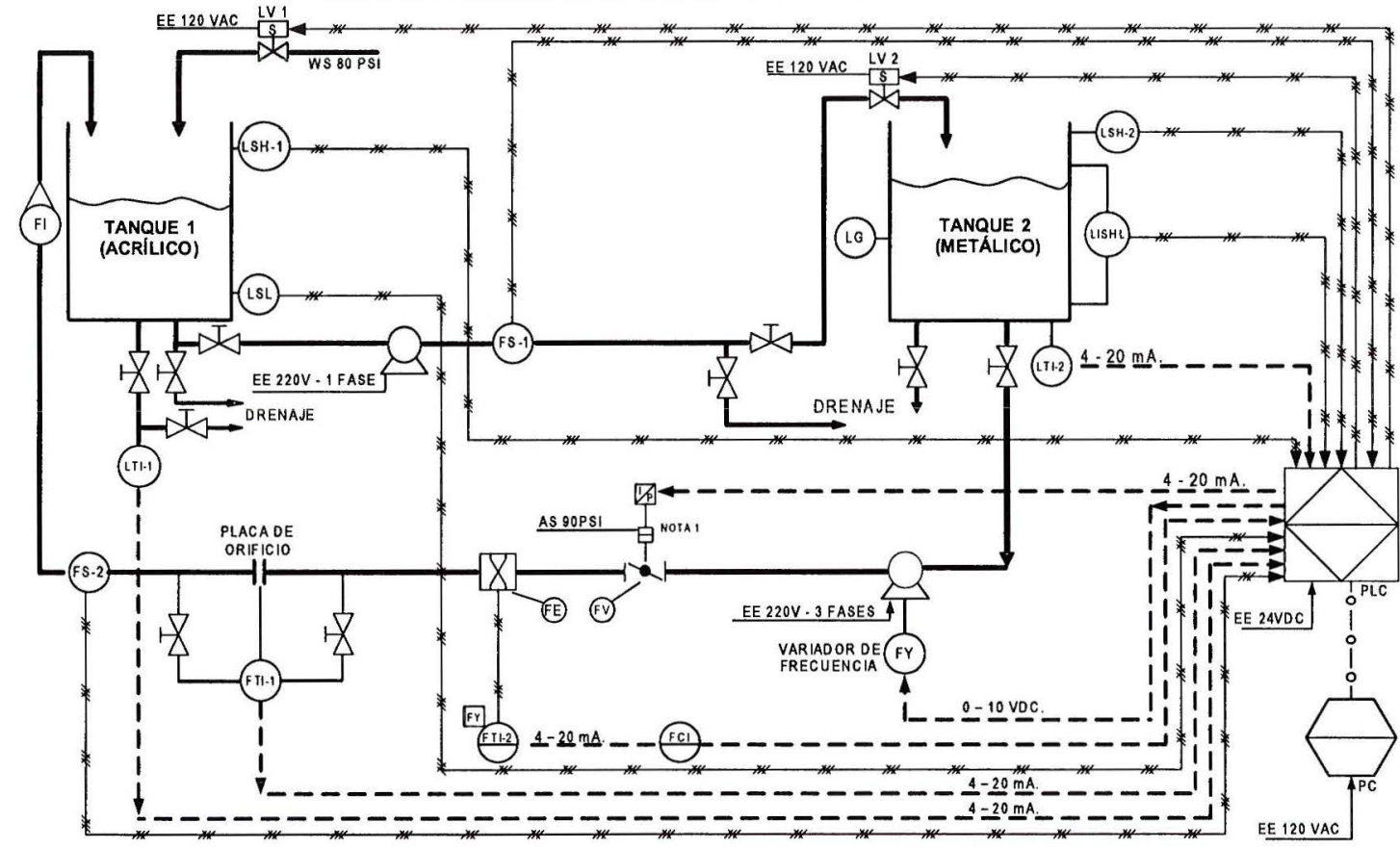

** Posibilidad de hacer control, con sólo modificar las conexiones en el tablero de control.

\begin{tabular}{|c|c|c|c|c|c|c|}
\hline 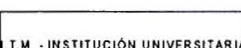 & 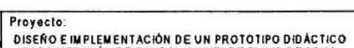 & Roalizado por: CML & Feens & Moditicaciones & Fostas dol & $2007 \cdot 01 \cdot 30$ \\
\hline & 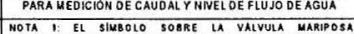 & Aprobado por: JLR. & & & & \\
\hline MEOELIN & 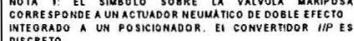 & Rovisado por: EEt & & & Hоа & 11 \\
\hline
\end{tabular}




\section{Referencias}

GUTIÉRREZ GARCÍA, Luis. Teoría de la medición de caudales y volúmenes de agua e instrumental necesario disponible en el mercado. Jefe Laboratorio de Agua. Centro Español de Metrología. 2003.

GRUPO TAR. Instrumentación y Control de Procesos, Módulo 3. [En línea]. Grupo TAR - Universidad de Sevilla, España. 2003. < <http://tar5.eup. us.es/master/ponencias/pdf/ic01a.pdf> (Fecha de consulta 12 de febrero de 2007). 69p.

ICA. Presión como Variable de Estado. [En línea]. ICA - Control y automatización. <http://www.geocities.com/CollegePark/Pool/1549/frame.html> [s.n]: [s.c] (Fecha de consulta 07 de abril de 2007).

KAUFFMAN D. Flow Sheets and Diagrams. University of New Mexico, Albuquerque, New Mexico. Module G1.5. p38 - 46. 1998.

M SQUARED LTD. Medición de nivel en líquidos $I$ y II. Impreso en la editorial de la Universidad Nacional de Colombia. 1990.

Medición de caudal en líquidos. Impreso en la editorial de la Universidad Nacional de Colombia. 1990.

ORDÓN̄EZ ARIAS, José Alberto; TREJOS NIETO, Diego Andrés. Medidores de flujo (flujómetros, Mecánica de fluidos e hidráulica de tuberías. Universidad Francisco de Paula Santander. Facultad de ingeniería civil. 2.005 .

SCHEER, John E. The basic of rotameters. [En línea]. Variable Area Flowmeters División, $\mathrm{ABB}$, Inc. <http://www.abbinfozone.com/> [s.n]: [s.c] (Fecha de consulta 11 de abril de 2007).

THE INTERNATIONAL SOCIETY FOR MEASUREMENT AND CONTROL. Autor Corporativo. American National Standard, Instrumentation Symbols and Identification. Norma ANSI/ISA-S5.1-1984 (R 1992) $122 p$. 
